\title{
Nonlinear interactions between kinetic Alfvén and ion-sound waves
}

\author{
G. Brodin, L. Stenflo and P. K. Shukla
}

Department of Physics, Umeå University, SE-901 87 Umeå, Sweden

\begin{abstract}
The resonant interaction between kinetic Alfvén and ion-acoustic waves is considered using the Hall-MHD theory. The results of previous authors are generalized to cover both finite Larmor radius as well as the ideal MHD results. It is found that the three wave coupling is strongest when the wavelength is comparable to the ion-sound gyroradius. Applications of our work to weak turbulence theories as well as to the heating of the solar corona are pointed out.
\end{abstract}


Keywords: Kinetic Alfvén waves, ion-sound waves, three-wave interactions, Hall-MHD equations, solar corona

The nonlinear interaction of magnetohydrodynamic (MHD) waves has been considered by numerous authors (see for example, Sagdeev and Galeev, 1969; Petviashvili and Pokhotelov, 1992; Shukla and Stenflo, 1999). The applications involve fusion plasmas (Hasegawa and Uberoi, 1982), space physics (Petviashvili and Pokhotelov, 1992; Shukla and Stenflo, 1999; Wu and Chao, 2004) as well as solar physics (Shukla et al., 1999; Voitenko and Goossens, 2000 and 2002; Shukla and Stenflo, 2005; Chandran 2005) and astrophysics (Ng and Bhattacharjee, 1996; Goldreich and Sridhar, 1997). The classic work on three wave interaction of ideal MHD waves (Sagdeev and Galeev, 1969) was later generalized to account for arbitrary (but still ideal) MHD wave modes and directions of propagations (Brodin and Stenflo, 1988). The ideal MHD processes were soon suggested to have applications for the heating of fusion plasmas (Lashmore-Davies and Ong, 1974). Hasegawa and Chen (1976a) showed, however, that processes involving kinetic Alfvén waves were more efficient for that purpose. The latter waves can be described by the Hall-MHD theory, and general three wave coupling coefficients for the Hall-MHD plasmas were thus deduced by Brodin and Stenflo (1990). Applications for the parametric decay instability of magneto-acoustic waves into two kinetic Alfvén waves, to the heating of the solar corona, were considered by Voitenko and Goossens (2002). The Joule electron heating caused by high-frequency dispersive Alfvén waves in the solar corona was also analysed by Shukla et al. (1999).

Much of the previous work describing parametric instabilities involving kinetic Alfvén waves (KAWs) has adopted a kinetic theory (Hasegawa and Chen, 1976; Voitenko 1998) or multi-fluid models (Erokhin, Moiseev and Mukhin, 1978; Voitenko and Goossens, 2002). In the present paper, we will however demonstrate that the essential characteristics of the three-wave decay interaction involving the KAWs can be more simply described within a unified formalism of the Hall-MHD theory. An important result of that formalism is that the decay of kinetic Alfvén waves is dominated by the excitation of modes with short perpendicular wavelengths, of the order of the ion-sound gyroradius, that must be described by the Hall-MHD theory. We shall show that this specific example has general significance, and that the ideal MHD typically is unable to deal with the nonlinear evolution of the MHD waves, even if the initial conditions are within the range of the ideal MHD.

Thus, we start with the Hall-MHD equations, that can be written as

$$
\begin{gathered}
\frac{\partial \rho}{\partial t}+\nabla \cdot(\rho \mathbf{v})=0, \\
\rho \frac{d \mathbf{v}}{d t}=-c_{s}^{2} \nabla \rho+\frac{(\nabla \times \mathbf{B}) \times \mathbf{B}}{\mu_{0}},
\end{gathered}
$$

and

$$
\frac{\partial \mathbf{B}}{\partial t}=\nabla \times\left(\mathbf{v} \times \mathbf{B}-\frac{m_{i}}{e} \frac{d \mathbf{v}}{d t}\right),
$$

where $d / d t=\partial / \partial t+\mathbf{v} \cdot \nabla, e$ and $m_{i}$ is the ion charge and mass, whereas $\rho, \mathbf{v}$, and $\mathbf{B}$ are the density, velocity and magnetic field, respectively, and $c_{s}=\left[\left(T_{e}+T_{i}\right) / m_{i}\right]^{1 / 2}$ is the ion-sound speed. Here $T_{e}$ and $T_{i}$ are the electron and ion temperatures.

Considering the resonant interaction between three waves which satisfy the matching conditions

$$
\omega_{3}=\omega_{1}+\omega_{2}
$$


and

$$
\mathbf{k}_{3}=\mathbf{k}_{1}+\mathbf{k}_{2},
$$

we can, using (1)-(5), derive the equations [see Brodin and Stenflo (1990) for details]

$$
\left(\frac{\partial}{\partial t}+\mathbf{v}_{g 1,2} \cdot \nabla\right) \rho_{1,2}=-\frac{1}{\partial \tilde{D}_{1,2} / \partial \omega_{1,2}} C \rho_{2,1}^{*} \rho_{3}
$$

and

$$
\left(\frac{\partial}{\partial t}+\mathbf{v}_{g 3} \cdot \nabla\right) \rho_{3}=\frac{1}{\partial \tilde{D}_{3} / \partial \omega_{3}} C \rho_{1} \rho_{2}
$$

where

$$
\begin{aligned}
& C=\frac{\omega_{1} \omega_{2} \omega_{3}}{\rho_{0} k_{1 \perp}^{2} k_{2 \perp}^{2} k_{3 \perp}^{2}} {\left[\frac{\mathbf{K}_{3} \cdot \mathbf{K}_{2}^{*}}{\omega_{1}} k_{1 \perp}^{2}+\frac{\mathbf{K}_{3} \cdot \mathbf{K}_{1}^{*}}{\omega_{2}} k_{2 \perp}^{2}+\frac{\mathbf{K}_{1}^{*} \cdot \mathbf{K}_{2}^{*}}{\omega_{3}} k_{3 \perp}^{2}-\right.} \\
& \frac{k_{1 \perp}^{2} k_{2 \perp}^{2} k_{3 \perp}^{2}}{\omega_{1} \omega_{2} \omega_{3}} c_{s}^{2}+\frac{i \omega_{c i}}{\omega_{3}}\left(\frac{k_{2 z}}{\omega_{2}}-\frac{k_{1 z}}{\omega_{1}}\right)\left(( \mathbf { K } _ { 3 } + \frac { i \omega _ { 3 } \mathbf { k } _ { 3 } \times \mathbf { K } _ { 3 } } { \omega _ { c i } k _ { 3 z } } ) \cdot \left(\mathbf{K}_{1}^{*}-\right.\right. \\
&\left.\left.\left.\frac{i \omega_{1} \mathbf{k}_{1} \times \mathbf{K}_{1}^{*}}{\omega_{c i} k_{1 z}}\right) \times\left(\mathbf{K}_{2}^{*}-\frac{i \omega_{2} \mathbf{k}_{2} \times \mathbf{K}_{2}^{*}}{\omega_{c i} k_{2 z}}\right)-\mathbf{K}_{3} \cdot\left(\mathbf{K}_{1}^{*} \times \mathbf{K}_{2}^{*}\right)\right)\right] \\
& \tilde{D}_{j}=\left[\omega_{j}^{4}-\omega_{j}^{2} k_{j}^{2}\left(c_{A}^{2}+c_{s}^{2}\right)+k_{j z}^{2} k_{j}^{2} c_{A}^{2} c_{s}^{2}\right. \\
&\left.-\frac{\left.\omega_{j}^{2} k_{j z}^{2} k_{j}^{2}\left(\omega_{j}^{2}-k_{j}^{2} c_{s}^{2}\right) c_{A}^{4}\right]\left(\omega_{j}^{2}-k_{j}^{2} c_{s}^{2}\right)}{\omega_{c i}^{2}\left(\omega_{j}^{2}-k_{j z}^{2} c_{A}^{2}\right)}\right]
\end{aligned}
$$

and

$$
\mathbf{K}_{j}=\mathbf{k}_{j \perp} \frac{\left(\omega_{j}^{2}-k_{j z}^{2} c_{s}^{2}\right)}{\omega_{j}^{2}}+\frac{i \hat{\mathbf{z}} \times \mathbf{k}_{j \perp}\left(\omega_{j}^{2}-k_{j}^{2} c_{s}^{2}\right) k_{j z}^{2} c_{A}^{2}}{\omega_{c i} \omega_{j}\left(\omega_{j}^{2}-k_{j z}^{2} c_{A}^{2}\right)}+\frac{k_{j \perp}^{2} k_{j z} c_{s}^{2}}{\omega_{j}^{2}} \hat{\mathbf{z}} .
$$

Here $\mathbf{v}_{g j}$ is the group velocity of wave $j, \omega_{c i}$ is the ion gyrofrequency, and $c_{A}=\left(B_{0} / \mu_{0} \rho_{0}\right)^{1 / 2}$ is the Alfvén speed. The derivation of (6) and (7) is straightforward (Brodin and Stenflo, 1990). Our result has the significant advantage that the same coupling coefficient $C$ appears in both (6) and (17). This means that the Manley-Rowe relations are always satisfied. We could alternatively have used, instead of $\rho_{j}$, longitudinal and/or transverse components of the velocity (using the relation $\left.\mathbf{v}_{j}=\rho_{j} \mathbf{K}_{j} \omega_{j} / k_{j \perp}^{2} \rho_{0}\right)$, where the transverse velocity component is particularly convenient for Alfvén waves with small or vanishing density perturbations.

Here, we focus on wave modes with frequencies well below the ion gyrofrequency, but with large perpendicular wavenumbers, so that $k_{\perp}^{2} c_{s}^{2} / \omega_{c i}^{2}$ can be of order unity. For the particular case of the KAWs, and for an intermediate beta plasma with $\left(m_{e} / m_{i}\right) c_{A}^{2}<c_{s}^{2} \ll c_{A}^{2}$, where $m_{e}$ is the electron mass, (10) can then be approximated by

$$
\mathbf{K} \approx-i \frac{\omega_{c i}}{\omega} \hat{\mathbf{z}} \times \mathbf{k}+\frac{k_{\perp}^{2} k_{z} c_{s}^{2}}{\omega^{2}} \hat{\mathbf{z}}
$$

(where the second term is smaller than the first by a factor of the order $c_{s} / c_{A}$ ), together with the approximate dispersion relation

$$
\omega^{2}=k_{z}^{2} c_{A}^{2}\left[1+\frac{k_{\perp}^{2} c_{s}^{2}}{\omega_{c i}^{2}}\right]
$$


Similarly, for the the ion-acoustic waves we can write

$$
\mathbf{K} \approx \frac{i k_{\perp}^{2} c_{s}^{2} \hat{\mathbf{z}} \times \mathbf{k}}{\omega_{c i} \omega}+\frac{k_{\perp}^{2} k_{z} c_{s}^{2}}{\omega^{2}} \hat{\mathbf{z}}
$$

provided that $\omega^{2} / \omega_{c i}^{2} \ll k_{z} / k_{\perp}$. The corresponding dispersion relation can then be approximated as

$$
\omega^{2}=\frac{k_{z}^{2} c_{s}^{2}}{\left(1+k_{2 \perp}^{2} c_{s}^{2} / \omega_{c i}^{2}\right)} .
$$

Next, considering two waves (with index 1 and 3) to be kinetic Alfvén waves described by (12), and one wave (with index 2) to be an ion-acoustic wave, described by (14), the interaction equations can be rewritten as

$$
\begin{aligned}
& \left(\frac{\partial}{\partial t}+\mathbf{v}_{g 1} \cdot \nabla\right) v_{1}=-\frac{i \omega_{1} C_{A m A} \rho_{2}^{*} v_{3}}{\rho_{0}} \\
& \left(\frac{\partial}{\partial t}+\mathbf{v}_{g 2} \cdot \nabla\right) \rho_{2}=i \frac{\rho_{0} k_{2 z}^{2}}{\omega_{2}} C_{A m A} v_{1}^{*} v_{3}
\end{aligned}
$$

and

$$
\left(\frac{\partial}{\partial t}+\mathbf{v}_{g 3} \cdot \nabla\right) v_{3}=-\frac{i \omega_{3} C_{A m A} v_{1} \rho_{2}}{\rho_{0}}
$$

with the coupling coefficient approximated by

$$
C_{A m A}=\cos \theta-\frac{\omega_{2}^{2} k_{1 \perp} k_{3 \perp}}{k_{2}^{2} c_{A}^{2} k_{1 z} k_{3 z}} \sin ^{2} \theta-\mathrm{i} \frac{c_{s}^{2} \sin \theta}{k_{2 z} \omega_{c i}}\left(\frac{k_{3 z}}{\omega_{3}}-\frac{k_{1 z}}{\omega_{1}}\right)\left[k_{1 \perp}^{2}+k_{3 \perp}^{2}-k_{2 \perp}^{2}+\frac{k_{1 \perp}^{2} k_{3 \perp}^{2} c_{s}^{2}}{\omega_{c i}^{2}}\right]
$$

where $v_{1,3}$ is the magnitude of the velocity of waves 1 and 3 , respectively, and $\theta$ is the angle between $\mathbf{k}_{1 \perp}$ and $\mathbf{k}_{3 \perp}$ (or the angle between $\mathbf{v}_{1}$ and $\mathbf{v}_{3}$ when $\mathbf{k}_{1,3 \perp} \rightarrow 0$ ). The first two terms in (18) dominate for $k_{j \perp}^{2} c_{s}^{2} \ll \omega_{c i}^{2}$, and agree with the ideal MHD coupling coefficient of Brodin and Stenflo (1988) in the low-beta limit considered here. The third term, which dominates for large perpendicular wavenumbers, agrees with the coupling coefficient of Hasegawa and Chen (1976), which was derived using a kinetic approach. As a specific example, we let wave 3 be a pump wave. To demonstrate the importance of the second term in (18), we assume that all waves have large perpendicular wavenumbers, such that $k_{\perp}^{2} c_{s}^{2} / \omega_{c i}^{2} \sim 1$. Furthermore, to facilitate an order of magnitude estimate of (18) we let $\mathbf{k}_{1 \perp}$ and $\mathbf{k}_{3 \perp}$ be approximately perpendicular to each other. In this case, the magnitude of the third part of $C_{A m A}$ can be estimated as

$$
C_{A m A} \sim \frac{\omega_{c i}}{\omega_{3}} \gg 1
$$

which is much larger than the first two parts of $C_{A m A}$ accounted for by the ideal MHD, and which do not exceed unity. As a consequence, the growth rate $\Gamma_{\mathrm{HM}}$ into short perpendicular wavelength modes (with $k_{\perp}^{2} c_{s}^{2} / \omega_{c i}^{2} \sim 1$, described by the Hall-MHD theory) is larger than the growth rate $\Gamma_{\text {IM }}$ due to the ordinary ideal MHD modes by a factor $\Gamma_{\mathrm{HM}} / \Gamma_{\mathrm{IM}} \sim \omega_{c i} / \omega$. Thus, the increased coupling strength into short wavelength modes with perpendicular wavelengths of the order of the ion-sound gyroradius $\left(c_{s} / \omega_{c i}\right)$ affects the parametric decay processes significantly. This is very important as the wave cascade processes (Goldreich and Sridhar, 1997) of weak turbulence theories are based on 
the resonant three-wave coupling mechanism. Moreover, while the general features of such processes lead to a broadening of the frequency spectrum, and energy transfer towards lower frequencies, we note that the energy transfer will mainly occur in the direction of higher coupling strength, i.e. into modes with short perpendicular wavelengths. Thus, even for an initial turbulent spectrum well within the range of the ideal MHD, wave cascade processes will eventually lead to the excitation of short perpendicular wavelengths and the necessity to use the Hall-MHD rather than the ideal MHD.

Assuming that wave 3 is a pump wave with magnetic field magnitude $B_{3}$, and using the estimate $B_{3} \sim v_{3} B_{0} / c_{A}$, we find the growth rate

$$
\Gamma_{\mathrm{HM}} \sim \omega_{c i} \frac{B_{3}}{B_{0}}
$$

It should, however, be pointed out that the present decay channel for a KAW into an ion-acoustic wave and another KAW is not unique. Other decay channels that have been investigated for KAWs can compete with it (e.g. Voitenko and Goossens 2000; Onishchenko et. al. 2004). These processes can spread out the KAW spectrum and thus prevent the parametric decay into ion-acoustic waves. To find out the relative importance of the decay into ion-acoustic waves as compared to the above mentioned processes, we should therefore compare our estimate (20) with the growth rates $\Gamma_{A A}$ of Voitenko and Goossens (2000), and $\Gamma_{\text {JGR }}$ of Onishchenko et al. (2004). We then use the estimates $\Gamma_{A A} \sim 0.2 \omega_{3} k_{3}^{2} \rho_{i} B_{3} / k_{3 z} B_{0}$, where $\rho_{i}=\left(T_{i} / m_{i}\right)^{1 / 2} / \omega_{c i}$ is the ion Larmor radius, and $\Gamma_{\text {JGR }} \sim 2 \omega_{3} D k_{3}^{2} B_{3}^{2} / k_{3 z}^{2} B_{0}^{2}$, where $D$ is a factor of order unity (Onishchenko et al., 2004). Onishchenko et al. (2004) showed that $\Gamma_{\mathrm{JGR}}$ is smaller than $\Gamma_{A A}$ if $B_{3} / B_{0}$ is smaller than a factor of the order $k_{3 z} \rho_{i}$. A comparison between $\Gamma_{\mathrm{HM}}$ of the present paper and $\Gamma_{A A}$ reveals that $\Gamma_{\mathrm{HM}} / \Gamma_{A A} \sim 5 \omega_{c i} k_{3 z} / \omega_{3} k_{3}^{2} \rho_{i}$. Although the estimates above are very crude, they show that the process we consider in the present paper can be even more important than those of previous papers for a significant range of parameters.

To summarize, we have reconsidered the interaction of kinetic Alfvén and ion-acoustic waves using the Hall-MHD theory. In particular, the three wave equations involving the nonlinear coupling between two kinetic Alfvén waves and one ion-acoustic wave have been explicitly presented. The same coupling coefficient (18) appears in all these equations, implying that the Manley-Rowe relations are fulfilled. Furthermore, our coupling coefficient (18) includes both the ideal MHD results of Brodin and Stenflo (1988), and the effects due to the kinetic approach of Hasegawa and Chen (1976a), in a unified formalism. As can be seen from (18), the wave coupling is strongest for perpendicular wavelengths of the order of the ion-sound gyroradius. As has been argued above, this has important consequences for several processes, such as for the parametric decay instabilities and wave cascades in weak turbulence theories. Moreover, the formalism presented above is relevant for plasma particle energization in the solar corona by kinetic Alfvén waves. In the solar corona, a kinetic Alfvén pump wave can be excited by a linear transformation of an Alfvén surface wave in the neighbourhood of the resonance region (Hasegawa and Chen, 1976b). The mode converted kinetic Alfvén wave can then further decay into a daughter kinetic Alfvén wave and a dispersive ion sound wave, as described here. The nonlinearly excited kinetic Alfvén waves can attain large amplitudes and small perpendicular wavelengths (Hasegawa and Chen, 1976b), and they could therefore be our most efficient agents for energization of ions and electrons by kinetic Alfvén wave phase mixing and Joule heating (Ionson, 1978; Hasegawa and Uberoi, 1982; Shukla et al., 1994; Cramer, 2001), as well as for turbulent heating and particle-KAW interactions. 
This research was partially supported by the Swedish Research Council.

[1] G. Brodin and L. Stenflo, J. Plasma Phys. 39, 277 (1988).

[2] G. Brodin and L. Stenflo, Contrib. Plasma Phys. 30, 413 (1990).

[3] B. D. G. Chandran, Phys. Rev. Lett. 95, 265004 (2005).

[4] N. F. Cramer, The Physics of Alfvén Waves (Wiley-VCH, Berlin, 2001).

[5] N. S. Erokhin, S. S. Moiseev and V. V. Mukhin, Soviet J. Plasma Phys. 4, 656 (1978).

[6] P. Goldreich and S. Sridhar, Astrophys. J. 485, 680 (1997).

[7] A. Hasegawa and L. Chen, Phys. Rev. Lett. 36, 1362 (1976a).

[8] A. Hasegawa and L. Chen, Phys. Fluids 19, 1924 (1976b).

[9] A. Hasegawa and C. Uberoi, The Alfvén wave. DOE Review Series-Advances in Fusion Science and Engineering (U.S. Department of Energy, Washington D. C., 1982).

[10] J. A. Ionson, Astrophys. J. 226, 650 (1978).

[11] C. N. Lashmore-Davies and R. S. Ong, Phys. Rev. Lett. 32, 1172 (1974).

[12] C. S. Ng and A. Bhattacharjee, Astrophys. J. 465, 845 (1996).

[13] O. G. Onishchenko, O. A. Pokhotelov, R. Z. Sagdeev, L. Stenflo, R. A. Treumann and M. A. Balikhin, J. Geophys. Res. 109, A03306 (2004).

[14] V. I. Petviashvili and O. A. Pokhotelov, Solitary Waves in Plasmas and in the Atmosphere (Gordon and Breach, Philadelphia, 1992).

[15] R. Z. Sagdeev and A. A. Galeev, Nonlinear plasma theory (Benjamin, New York, 1969).

[16] P. K. Shukla, U. de Angelis, R. Bingham and L. Stenflo, (Eds.) Wave-Particle Interaction and Energization in Plasmas (Phys. Scripta, T50, 1994).

[17] P. K. Shukla, R. Bingham, J. F. McKenzie and W. I. Axford, Solar Phys. 186, 61 (1999).

[18] P. K. Shukla and L. Stenflo, in Nonlinear MHD waves and Turbulence, Ed. by T. Passot and P.-L. Sulem, (Springer, Berlin, 1999.), pp. 1-30.

[19] P. K. Shukla and L. Stenflo, Phys. Rev. Lett. 95, 269501 (2005).

[20] Y. M. Voitenko, J. Plasma Phys., 60, 497, (1978).

[21] Y. M. Voitenko and M. Goossens, Astron. Astrophys. 357, 1073 (2000)

[22] Y. M. Voitenko and M. Goossens, Solar Phys. 209, 37 (2002)

[23] D. J. Wu and J. K. Chao, Nonl. Proc. Geophys. 11, 631 (2004). 